\title{
Analysis of BWR Station Blackout Accident -Thermal-Hydraulic Behavior up to Severe Core Damage in Fukushima Daiichi Power Plant Unit 2-
}

\author{
Tadashi WATANABE ${ }^{1, *}$, Masahiro ISHIGAKI ${ }^{1}$, Akira SATO $^{1}$ \\ and Hideo NAKAMURA ${ }^{1}$
}

${ }^{1}$ Safety Research Center, Japan Atomic Energy Agency, 2-4 Shirakata Shirane, Tokai-mura, Naka-gun, Ibaraki 319-1195, Japan

\begin{abstract}
The analysis of the long-term station blackout accident of BWR has been performed using the TRAC-BF1 code. The actuation of RCIC (reactor core isolation cooling) was assumed, and the results were compared with the data observed at the Fukushima Daiichi Power Plant Unit 2 reactor. BWR-5 of 1,100 MW was analyzed, while the unit 2 reactor was BWR-4 of $780 \mathrm{MW}$. The reactor pressure and the core liquid level were, however, in good agreement with the observed data. It was confirmed that the quasi-steady state continued for a long time with the RCIC actuation. The timing of recovery action, which was composed of depressurization and coolant injection, necessary for the maximum clad temperature being less than 1,500 $\mathrm{K}$ was studied and compared with that of the unit 2 reactor.
\end{abstract}

KEYWORDS: BWR, station blackout, RCIC, TRAC-BF1, Fukushima Daiichi

\section{Introduction}

During the Great East Japan Earthquake on March 11th 2011, a station blackout accident ensued at the Fukushima Daiichi Nuclear Power Plant due to the earthquake and tsunami that followed. Although the emergency safety equipment was partially operational, reactor core damage occurred in Units 1 through 3. The detailed development of events is unclear, but major issues were presumably the loss of external power, loss of emergency power, and loss of cooling function due to partial operation of emergency safety equipment after the reactor scram due to seismic acceleration. This rapid communication presents the analysis and study results of the thermal-hydraulic behavior of Unit-2, which was the last to experience core damage among the three units, using the safety analysis code TRAC-BF $1^{1)}$ up until the time the reactor core damage occurred. To allow prompt reporting, the input model of Unit-2 itself (BWR4, 780,000 KW) was not used, and the input data for analysis of the existing 1,100,000 KW class BWR-5 were prepared instead ${ }^{2}$. Because a comparatively steady reactor core cooling status continued as a result of the actuation of the reactor core isolation cooling (RCIC) system during the station blackout in Unit-2, actuation of RCIC was assumed in the analysis while

\footnotetext{
* Corresponding author, E-mail: watanabe.tadashi66@jaea.go.jp

DOI : 10.15669 /fukushimainsights. Vol.4.46

(C) 2021 Atomic Energy Society of Japan. All rights reserved.

Originally published in Transactions of the Atomic Energy Society of Japan (ISSN 1347-2879), Vol. 10, No. 4, p.240-244

(2011) in Japanese. (Japanese version accepted: August 3, 2011)
} 
investigating the pressure reducing operation after stoppage of RCIC and the variation in reactor conditions before injection of cooling water. The analysis was justified by comparison with the observed data for Unit-2, including nuclear reactor pressure, etc ${ }^{3)}$. A sensitivity analysis was conducted adopting the timing of RCIC stoppage and start of the reactor core cooling function recovery operation as parameters and evaluating the effectiveness of operation.

\section{Analysis of BWR Station Blackout Accident}

\section{Analysis Conditions}

The node assignment used for the analysis is shown in Figure 1. The object reactor is a $1,100,000 \mathrm{KW}$ class BWR-5 ${ }^{2)}$, modeled from the water supply line to the main steam pipe. In the analysis, input data were prepared by setting up the thermal-hydraulic values acquired by steady state simulation as the initial condition, and assuming reactor scram due to seismic acceleration at time "zero," turbine trip, stoppage of recirculation pump, closure of the main steam isolation valve (MSIV), turbine bypass valve close and main water supply stop due to station blackout ${ }^{4}$. Among the emergency safety equipment, only the actuation of RCIC was assumed.

The rated design flow rate and operational start/stop conditions based on the water level of the nuclear reactor were specified ${ }^{2)}$ but the water level of the reactor core was kept stable for an extended period in Unit-2. Therefore, in the analysis, the flow rate of cooling water injected

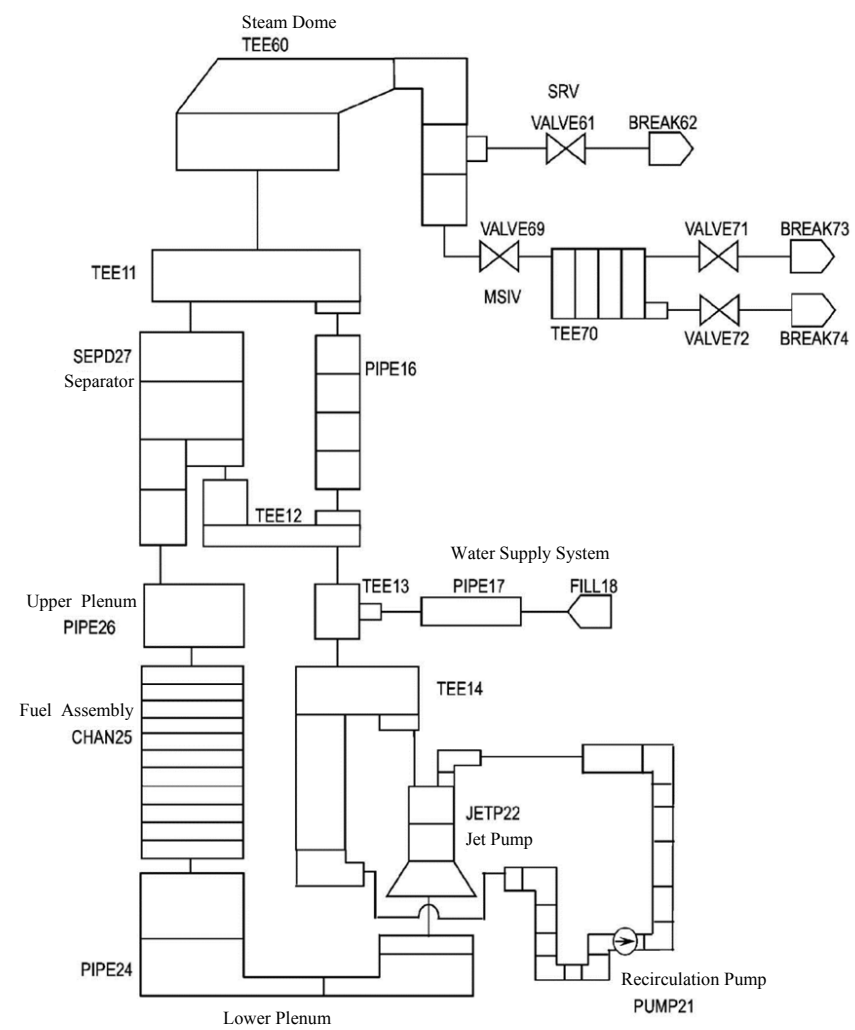

Figure 1 TRAC-BF1 nodding diagram for BWR analysis 
into the reactor core by the RCIC system and the extracted steam flow rate for driving the RCIC turbine were set up as boundary conditions to balance the nuclear reactor thermal output after the scram. The initial value was set to approximately $30 \mathrm{~kg} / \mathrm{s}$, and by gradual decrease the value fell to approximately $12 \mathrm{~kg} / \mathrm{s}$ after $50,000 \mathrm{~s}$, and approximately $10 \mathrm{~kg} / \mathrm{s}$ after 250,000 s. The line for VALVE72 was employed as the steam line for RCIC, and the steam flow rate was established by adjusting the opening of VALVE69 in the node assignment, as shown in Figure 1. The line for VALVE72 and VALVE69 were originally used to model the turbine bypass line and the MSIV, respectively, but because these were not used except for in the initial stage, they were used as substitutes for the steam lines for RCIC. The water supply line after stoppage of the main water line was directly employed for water injection. The water injection temperature was set to $300 \mathrm{~K}$ for the first $50,000 \mathrm{~s}$, then augmented to $373 \mathrm{~K}$. This is because the water source for water injection by the RCIC system was switched from the recovery tank at the initial stage to the suppression pool, but the temperature rise of the water source after switching was not taken into consideration.

Because the RCIC system is considered to have stopped at around 250,000 s for Unit-2, the operation up until then is assumed for the analysis as well. Afterwards, the injection quantity from the injection line and the extraction quantity from the steam line were both set to zero. The pressure reducing operation for the recovery of the cooling function in Unit-2 and the water injection by the fire engine was tried after 20,000 s. In the analysis, a water injection of $15 \mathrm{~kg} / \mathrm{s}$ was assumed at the time that pressure was reduced to approximately $0.6 \mathrm{MPa}$ by the pressure reducing operation after RCIC stop and these conditions were defined as the base case. The average water injection rate of Unit-2 was set up as the flow rate without considering differences in the reactor thermal output. The pressure reduction and water injection after RCIC stoppage was regarded as the reactor core cooling recovery operation. Furthermore, the time of operation start and the time RCIC stoppage were adopted as parameters for the sensitivity analysis, and the availability and effectiveness of the operation were studied.

\section{Comparison of Base Case and Unit-2}

The comparison of reactor pressure in the base case and observed data of Unit-2 are shown in Figure 2. The pressures from the analysis results are taken from the values for the steam

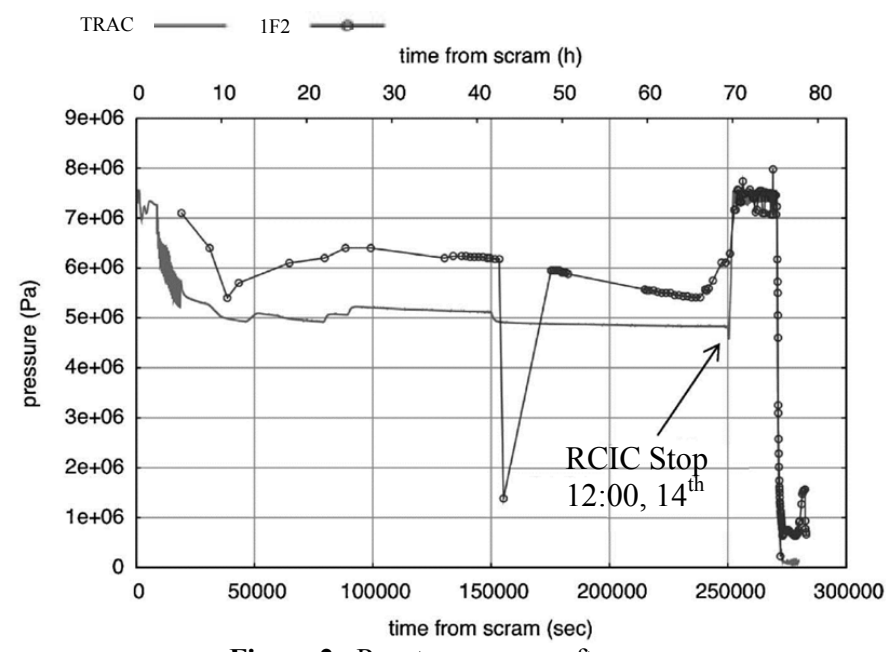

Figure 2 Reactor pressure after scram 
dome and depicted as solid lines. Furthermore, elapsed time after the scram is indicated in hours (h) in the upper part of the chart for comparison. The pressure increases slightly after the steady state of 7.0 MPa during the transition of events due to the blackout immediately after the scram, then gradually decreases accompanied by slight fluctuations, entering a stable condition at approximately $5 \mathrm{MPa}$ while wavering in a quasi-steady condition for approximately 200,000 s until the RCIC system ceases to function. The observed data indicated by circular symbols decrease from approximately $7 \mathrm{MPa}$ to $5.4 \mathrm{MPa}$ and show slight variations rising to approximately $6.4 \mathrm{MPa}$ and falling again to $5.4 \mathrm{MPa}$, exhibiting a quasi-steady condition during the period of $40,000 \mathrm{~s}$ to $240,000 \mathrm{~s}$. Accordingly, the analysis result portrays the nuclear reactor condition under the operation of RCIC rather well, even though the pressure is underestimated. In the analysis, RCIC stoppage was assumed to occur at 250,000 s, and the pressure rose due to the cutoff of the water supply and steam discharge to around the safety relief valve (SRV) setup pressure of 7.6 MPa. During this process, the steam generated in the reactor core was gradually discharged from the SRV. The pressure abruptly decreased at $270,000 \mathrm{~s}$ because the SRV was manually opened to reduce the pressure. The observed data exhibited a gradual increase of pressure from around 240,000 s, which was understood to indicate a gradual slowdown of RCIC and subsequent cessation, i.e., the speed of the turbine gradually slowed down and stopped within approximately $240,000-10,000 \mathrm{~s}$, causing the decrease in the steam flow rate. Then, an abrupt rise of pressure occurred as a result of the complete stop near $250,000 \mathrm{~s}$. Until the manual reduction of pressure, the pressure fluctuated during the SRV setup pressure, justifying the analysis. The pressure of Unit- 2 was reduced to the containment pressure of $0.6 \mathrm{MPa}$ at the time by manual reduction, while in the analysis the pressure reduced approximately to atmospheric pressure, as the increased pressure of containment was not taken into consideration.

A comparison of the reactor core water level of the study with the observed data (see Figure 3) shows them acting similarly to the pressure. For the purpose of comparison, the top of active fuel (TAF) was set to zero. In the analysis result, similarly to the pressure, an almost constant value of approximately $3.3 \mathrm{~m}$ was observed post-fluctuation after the scram and a quasi-steady state was realized until the RCIC stop, while exhibiting a gradual increase. The observed data showed a slightly higher value, but were stable until the RCIC stop, similarly to the analysis. This, likewise, demonstrates the justifiability of the analysis, i.e., in the analysis, the steam discharge and water supply quantities were specified in such a way that a balance with the

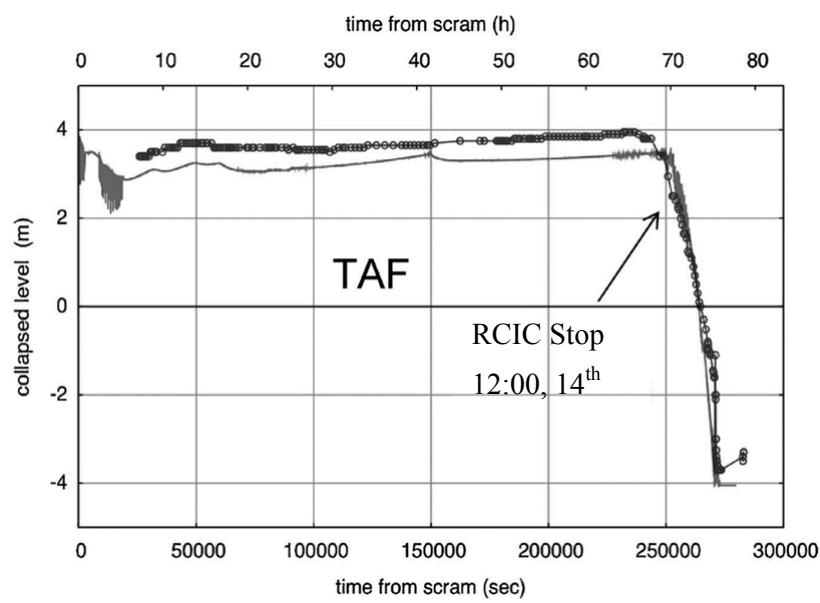

Figure 3 Core liquid level after scram 
reactor thermal output is maintained, and steady behavior without on/off of the RCIC seems to have taken place in Unit-2 as well. It is possible to adjust the analysis result to the observed data by finely calibrating the steam flow rate and water supply quantity. However, no further adjustment was made because it was considered that it would not greatly influence the development of events. In the analysis, a decrease in the water level was observed after the stoppage of RCIC at 250,000 s, while the observation data indicate a gradual decrease initiated before that. This can be explained by the water supply quantity starting to decrease due to the gradual slowdown of RCIC operation, with the decrease accelerating after a complete stop.

\section{Effect of Cooling Function Recovery Operation}

From the comparison of analysis and observed data, the development in the Unit-2 accident can be regarded as sufficiently replicated by this analysis, although the input data are different with respect to the reactor type and the power.

Furthermore, the assumption for the operation of RCIC can be regarded as being a reasonable one for achieving a stable reactor condition. For this reason, a sensitivity analysis was conducted with different times for RCIC stoppage and the reactor cooling recovery operation in the base case, and the resulting efficiency of operation was studied. The cooling recovery operation comprised two steps; pressure reduction by the SRV after a certain amount of time from the RCIC stop and the injection start when the pressure decreased to $0.6 \mathrm{MPa}$ by pressure reduction.

Mapping of the times of RCIC stop and the corresponding efficiency of operation is shown in Figure 4. The horizontal axis is the time of RCIC stop assuming that only the RCIC system was operational after the scram, and the vertical axis indicates the time from RCIC stop until the start of the cooling recovery operation. The symbol " $x$ " indicates the case where the peak fuel clad temperature in the reactor core is kept under $1,500 \mathrm{~K}$ and the symbol "+" indicates the case where the peak fuel clad temperature exceeds 1,500 K, i.e., the zone under the solid lines connecting these symbols can be regarded as the range in which the recovery operation is effectively functional after the RCIC system ceased to function. The solid lines indicate a gradual increase toward the right side with the lapse of time. This corresponds to a gradual increase in required time until operation start due to a decreased core output with respect to time.

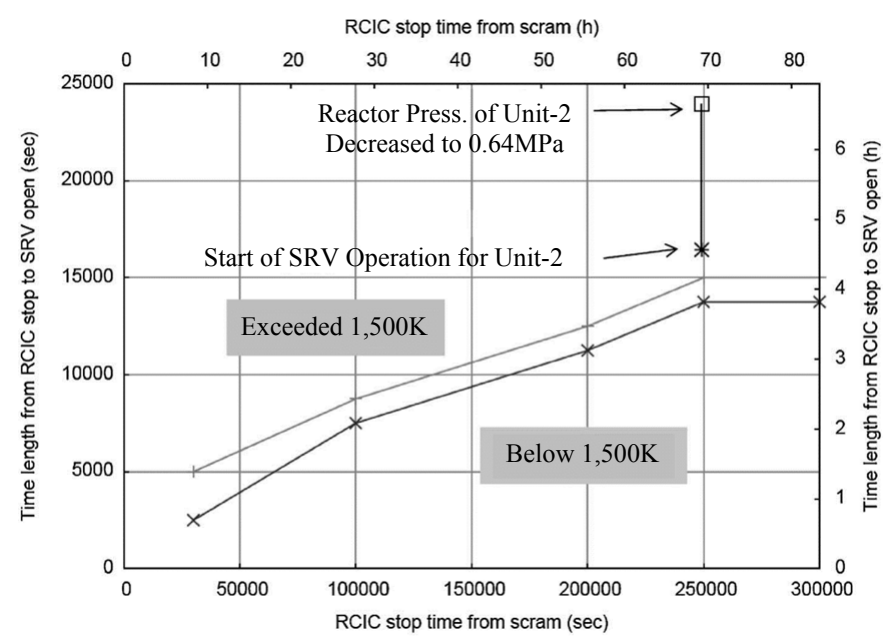

Figure 4 Timing map for cooling recovery operation 
Additionally, a band of time is observed on the boundary of the ranges where $1,500 \mathrm{~K}$ is exceeded and under it. This is caused by a change of several thousand seconds in the operation start time in the sensitivity analysis. Furthermore, 1,500 $\mathrm{K}$ was established as the value corresponding to the peak clad temperature of $1,200^{\circ} \mathrm{C}$ used in safety analyses. For completion at lower temperatures, an early operation start is required.

There is a possibility that the RCIC system stopped around 250,000 s in Unit-2, but for such a case, the chart implies that the recovery operation needs to start within $13,000 \mathrm{~s}$ after stoppage. The time when the pressure reduction operation actually started in Unit-2 and the time when the pressure decreased to $0.64 \mathrm{MPa}$ are shown for the purpose of comparison. The operation start time is in the slightly upper side of the range of analysis results, but if the assumption and errors in the analysis are considered, one can deduce that the timing is sensitive. However, some time before completion is required for actual pressure reduction after starting the operation, and thus the cladding tubes and fuels had already reached high temperatures by the time water injection began.

\section{Conclusions}

An analysis of the station blackout accident of a BWR was conducted over an extended time period using the TRAC-BF1 code. Only the RCIC operation was assumed. Comparison was made with observed data from Unit-2 of the Fukushima Daiichi Nuclear Power Plant, and the effectiveness of the cooling recovery operation after the RCC stop was evaluated. The analysis was conducted on a different 1,100,000 KW class BWR-5, but both the pressure of the nuclear reactor and the reactor water level showed good agreement with the data of the 780,000 KW BWR-4, in Unit-2 of the power plant. We confirmed that a quasi-steady thermal-hydraulic condition was preserved for an extended period of time. Furthermore, the necessary timing for starting the required operation in order to keep the peak clad temperature under $1,500 \mathrm{~K}$ was studied in the case of conducting pressure reduction and the subsequent injection of cooling water as steps in the cooling recovery operation after RCIC stop, and a comparison was made with the case of Unit-2.

We would like to express our gratitude to Dr. Masashi Hirano, the director of the Nuclear Safety Research Center, who gave us valuable suggestions and comments during the preparation of this rapid communication.

\section{References}

1) M. M. Giles, G. A. Jayne, S. Z. Rouhani et al., TRAC-BF1/MOD1: An Advanced Best-Estimate Computer Program for BWR Accident Analysis, NUREG/CR-4356 (1992).

2) J. Ishikawa, K. Muramatsu, T. Sakamoto, Systematic Source Term Analysis for Level 3 PSA of a BWR with Mark-II Type Containment with THALES-2 Code, JAERI-Research 2005-021, Japan Atomic Energy Research Institute (JAERI) (2005). [in Japanese]

3) http://www.tepco.co.jp, Tokyo Electric Power Co. (TEPCO) (2011).

4) D. D. Yue, W. A. Condon, Severe-Accident-Sequence Assessment of Hypothetical Complete Station Blackout at the Browns Ferry Nuclear Plant, CONF-810905-9 (1981). 